\title{
A REALIDADE VIRTUAL NA REABLITAÇÃO DE EXTREMIDADES SUPERIORES PÓS ACIDENTE VASCULAR CEREBRAL: UMA REVISÃO INTEGRATIVA.
}

\author{
Alexssander Weber Crivellaro'; Brenda Souza Severo²; Carolina Favero \\ Kerche $^{3}$; Natália Dal Forno4; Larissa Gasparini da Rocha5; Nathaly Marin \\ Hernandez ${ }^{6}$
}

\section{RESUMO}

Objetivo: Investigar os efeitos da Realidade Virtual na reabilitação fisioterapêutica de pacientes com comprometimento no membro superior em decorrência do Acidente Vascular Cerebral. Metodologia: Estudo do tipo revisão de literatura integrativa, selecionados artigos das bases de dados PubMed e Scielo. Critérios de inclusão: Ensaios clínicos dos últimos cinco anos, língua inglesa, que possuíssem como tema o uso da realidade virtual na reabilitação de pacientes pós Acidente Vascular Cerebral. Critérios de exclusão: Manuscritos não disponíveis na íntegra de forma gratuita, revisões, outras doenças neurológicas, estudos incompletos, participação em testes de projetos, desenvolvimento de programas de Realidade Virtual e estudos com outro método como reabilitação principal. Resultados: Selecionados 36 artigos após a pesquisa, e após a aplicação dos critérios de exclusão restaram 7 artigos. Conclusão: Em relação a funcionalidade de membros superiores, a Realidade Virtual é considerada um método viável e seguro na reabilitação dos pacientes pós Acidente Vascular Cerebral.

Palavras Chaves: Tecnologia Virtual; Acidente Cerebrovascular; Técnicas Fisioterápicas.

Eixo Temático: Atenção Integral e Promoção à Saúde (AIPS).

\section{INTRODUÇÃO}

De acordo com a Organização Mundial da Saúde (2020) o acidente vascular cerebral (AVC) é uma doença crônica não transmissível caracterizada como a $2^{\circ}$ principal causa de morte no mundo, correspondendo a um total de $11 \%$ das

\footnotetext{
${ }_{1}^{1}$ Alexssander Weber Crivellaro - Universidade Franciscana alexcrivellaro2@gmail.com

2 Brenda Souza Severo - Universidade Franciscana brenda.s.severo@gmail.com

${ }^{3}$ Carolina Favero Kerche - Universidade Franciscana carolinakerche99@gmail.com

${ }^{4}$ Natália Dal Forno - Universidade Franciscana natalia.dforno@ufn.edu.br

${ }^{5}$ Larissa Gasparini da Rocha -Universidade Franciscana larissa.rocha@ufn.edu.br

${ }^{6}$ Nathaly Marin Hernandez - Universidade Franciscana nathaly.hernandez@ufn.edu.br
} 
mortalidades. Este é uma doença que possui origem vascular, sendo classificado em isquêmico quando ocorre uma interrupção do fluxo sanguíneo cerebral em decorrência de uma obstrução, e hemorrágico que surge em função do rompimento de um vaso, causando uma hemorragia intracerebral (MINISTÉRIO DA SAÚDE, 2013).

Diversos são os fatores de risco que podem desencadear a doença, alguns não modificáveis como as doenças hereditárias, etnia, sexo, idade, baixo peso ao nascer, dentro outros, e, os fatores de risco considerados modificáveis como o tabagismo, diabetes mellitus, hipertensão, síndrome metabólica, uso indiscriminado de álcool e drogas, doenças do coração, dislipidemia e reposição hormonal (SIQUEIRA; SCHNEIDERS; SILVA, 2019).

Estima-se que tal condição acometa em maior parte a população adulta, sendo responsável por um grave problema de saúde pública, visto que ele enquadra-se como a primeira causa de perda da funcionalidade na realização de atividades de vida diárias. Assim, gerando um impacto negativo na funcionalidade, e consequentemente acarretar em danos na qualidade de vida dos indivíduos acometidos (RANGEL; BELASCO; DICCINI, 2013).

O AVC é um grande desafio ao longo do processo de reabilitação, seja para o indivíduo acometido, quanto ao cuidador, de maneira que seja necessária a realização de uma reabilitação específica para que o paciente possa obter ganhos em sua funcionalidade e recuperá-la com o máximo de qualidade possível (RAMOSLIMA et al., 2018). Diante do exposto, o tratamento consiste na prevenção através de mudanças nos hábitos diários para que se tenha um estilo de vida saudável, e na reabilitação através da fisioterapia, sendo imprescindível haver uma abordagem multiprofissional durante esse processo de reparação (SIQUEIRA; SCHNEIDERS; SILVA, 2019).

Um dos métodos terapêuticos muito utilizados na reabilitação fisioterapêutica do $A V C$ é a realidade virtual (RV) que oferece uma conexão entre o usuário e o sistema, através de dispositivos multissensoriais, sendo possível executar a prática de atividades nesse cenário, através de movimentos das mãos e corpo, ou com o uso de dispositivos táteis como luvas, joystics e mouse (BARILLI; EBECKEN; 
CUNHA, 2011; ARAMAKI et al., 2019).

Desta maneira, a escolha do tema se justifica em investigar de que forma os pacientes acometidos por um AVC podem obter melhoras em relação a sua funcionalidade e independência através do uso da RV durante o processo de reabilitação. Espera-se que tal método traga diversos benefícios ao paciente de forma que ele possa melhorar a sua saúde e bem estar.

Como forma de e lucidar o uso da RV e auxiliar na maior compreensão sobre o tema, o estudo busca responder o seguinte problema de pesquisa: Quais os efeitos da RV nos membros superiores acometidos de pacientes pós AVC? $O$ presente estudo tem o objetivo de investigar quais são os efeitos da aplicação da RV durante 0 período de reabilitação fisioterapêutica em pacientes com comprometimento no membro superior em decorrência do AVC.

\section{METODOLOGIA}

O estudo apresentado trata-se de uma revisão de literatura do tipo integrativa, onde foi realizada uma seleção de estudos dos últimos cinco anos nas bases de dados PubMed (National Library of Medicine) e Scielo (Scientific Electronic Library Online), através dos descritores Realidade Virtual, Acidente Vascular Cerebral e Fisioterapia, todos utilizados na língua inglesa e com o uso do operador booleano AND entre os descritores citados. Os artigos selecionados para o estudo seguiram os determinados critérios de inclusão: ensaios clínicos dos últimos cinco anos publicados entre 2016 a 2021 na língua portuguesa e inglesa,que possuíssem como tema o uso da RV na reabilitação de pacientes pós AVC e com o objetivo voltado para o acometimento dos membros superiores. Em relação aos critérios de exclusão se enquadram manuscritos que não estavam disponíveis na íntegra de forma gratuita, as revisões sistemáticas, outras doenças neurológicas, estudos incompletos, participação em testes de projetos, desenvolvimento de programas de realidade virtual e estudos que citassem outro método como reabilitação principal.

\section{RESULTADOS}




\section{QUFN}

Através desta pesquisa, 36 publicações foram encontradas, em que 29 dos resultados pertenceram a base de dados PubMed, onde 23 artigos foram excluídos, restando 6 artigos selecionados. A base de dados Scielo correspondeu a um total de 7 artigos, sendo que apenas 1 se enquadrou nos critérios de inclusão. Um total de 7 artigos foram selecionadosneste estudo.

Figura 1- Fluxograma do processo de exclusão.

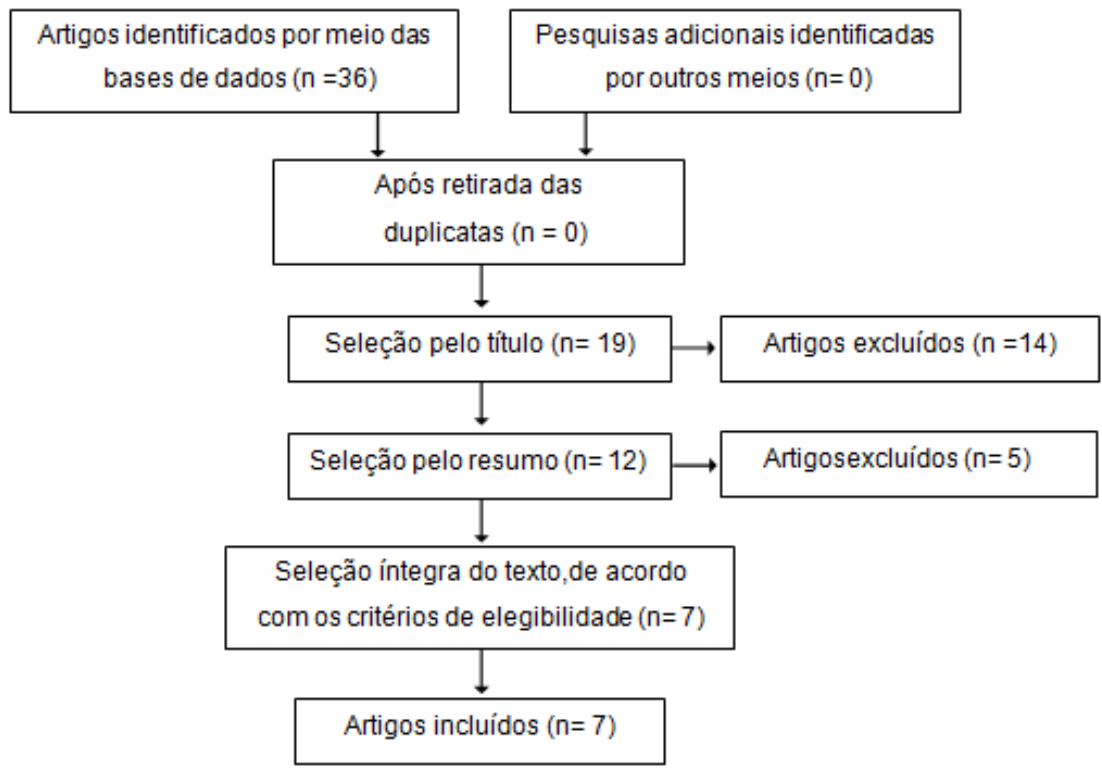

Fonte: autores. 
Quadro 1 - Artigos selecionados.

\begin{tabular}{|c|c|c|c|c|}
\hline Autor/ano & Objetivo & Amostra & Resultado & Conclusão \\
\hline $\begin{array}{l}\text { Norousi-Gheidari, N. et } \\
\text { al. (2020) }\end{array}$ & $\begin{array}{l}\text { Avaliar a segurança e } \\
\text { viabilidade do sistema } \\
\text { de exergaming de } \\
\text { reabilitação e fornecer } \\
\text { evidências } \\
\text { preliminares sobre a } \\
\text { sua eficácia clínica na } \\
\text { recuperação funcional } \\
\text { de extremidade } \\
\text { superior pós AVC. }\end{array}$ & $\begin{array}{l}18 \text { indivíduos que } \\
\text { sofreram um AVC e } \\
\text { com déficit motor de } \\
\text { extremidade superior. }\end{array}$ & $\begin{array}{l}\text { As medidas de eficácia mostraram } \\
\text { melhoras estatisticamente significativas } \\
\text { nas atividades de vida diárias e os } \\
\text { domínios de mobilidade e físico do Stroke } \\
\text { Impact Scale (SIS) no pós intervenção. }\end{array}$ & $\begin{array}{l}\text { O uso do sistema de exergaming de } \\
\text { forma suplementar na reabilitação de } \\
\text { pacientes com AVC é viável e seguro } \\
\text { podendo ser benéfico na recuperação da } \\
\text { função dos membros superiores. }\end{array}$ \\
\hline Oh,Y. et al. (2019) & $\begin{array}{l}\text { Investigar se a RV } \\
\text { combinada } \\
\text { instrumentos reais são } \\
\text { eficientes na melhora } \\
\text { da função de membro } \\
\text { superior e função } \\
\text { cognitiva em pacientes } \\
\text { pós AVC. }\end{array}$ & $\begin{array}{l}31 \text { pacientes com AVC } \\
\text { crônico (> 6meses) } \\
\text { com disfunção leve a } \\
\text { moderada de } \\
\text { membros superiores. }\end{array}$ & $\begin{array}{l}\text { Maiores benefícios foram obtidos no } \\
\text { grupo experimental em relação a potência } \\
\text { motora de extensão de punho, } \\
\text { espasticidade de cotovelo, testes } \\
\text { realizados, força de pinça lateral, palmar } \\
\text { e ponta, antes e imediatamente o treino. }\end{array}$ & $\begin{array}{l}\text { O treinamento de RV combinado com } \\
\text { instrumentos reais foi eficaz na melhora } \\
\text { da capacidade funcional das } \\
\text { extremidades superiores em pacientes } \\
\text { crônicos devido ao AVC. }\end{array}$ \\
\hline $\begin{array}{llll}\text { Ogun,M N. } & \text { et } & \text { al. } \\
(2019) & & & \end{array}$ & $\begin{array}{l}\text { Investigar a eficácia da } \\
\text { RV imersiva na função } \\
\text { de } \quad \text { extremidade }\end{array}$ & $\begin{array}{l}65 \text { pacientes com } \\
\text { AVC isquêmico }(<6 \\
\text { meses). }\end{array}$ & $\begin{array}{l}\text { Ocorreram melhoras significativas no } \\
\text { grupo de RV em relação a funcionalidade, } \\
\text { independência funcional e habilidades de }\end{array}$ & $\begin{array}{l}\text { Aplicações imersivas de RV impactam } \\
\text { positivamente na reabilitação da função } \\
\text { de membros superiores e atividades de }\end{array}$ \\
\hline
\end{tabular}




\begin{tabular}{|c|c|c|c|c|}
\hline & $\begin{array}{l}\text { superior em pacientes } \\
\text { com AVC isquêmico. }\end{array}$ & & $\begin{array}{l}\text { autocuidado, quando comparados ao } \\
\text { grupo controle. }\end{array}$ & $\begin{array}{l}\text { vida diária, mas que não melhoram a } \\
\text { independência nos pacientes com AVC. }\end{array}$ \\
\hline $\begin{array}{l}\text { Lee, M M; Lee, } \mathrm{K} \text { J; } \\
\text { Song, C H. (2018) }\end{array}$ & $\begin{array}{l}\text { Investigar os efeitos } \\
\text { do treinamento de RV } \\
\text { de remo em canoa } \\
\text { baseado em jogos, } \\
\text { quando combinados } \\
\text { com programas } \\
\text { convencionais de } \\
\text { reabilitação física, no } \\
\text { equilíbrio postural e } \\
\text { função de membros } \\
\text { superiores em } 30 \\
\text { pacientes com AVC } \\
\text { subagudo. }\end{array}$ & $\begin{array}{l}30 \text { pacientes com } \\
\text { AVC subagudo }(<6 \\
\text { meses }) .\end{array}$ & $\begin{array}{l}\text { Após cinco semanas, o } \\
\text { experimental } \\
\text { significativamente maior no } \\
\text { postural, função dos membros superiores, } \\
\text { estabilidade de tronco no lado afetado e } \\
\text { do lado não afetado e oscilação postural, } \\
\text { quando comprado ao grupo controle. }\end{array}$ & $\begin{array}{l}\text { O treino de remo em canoa através da } \\
\text { RV baseado em jogos pode ser mais } \\
\text { eficaz que a reabilitação convencional } \\
\text { sozinha na melhora do equilíbrio postural } \\
\text { e função dos membros superiores nos } \\
\text { pacientes com AVC subagudo. }\end{array}$ \\
\hline $\begin{array}{l}\text { Perez-Marcos, D. et al. } \\
\text { (2017) }\end{array}$ & $\begin{array}{l}\text { Investigar a viabilidade } \\
\text { de aplicação do } \\
\text { treinamento intensivo } \\
\text { em pacientes com } \\
\text { AVC crônico utilizando } \\
\text { a RV. }\end{array}$ & $\begin{array}{l}10 \text { pacientes com AVC } \\
\text { crônico (> } 6 \text { meses) } \\
\text { com hemiparesia de } \\
\text { extremidade superior. }\end{array}$ & $\begin{array}{l}\text { Os pacientes apresentaram altos níveis } \\
\text { de função com melhora significativa na } \\
\text { função motora pós intervenção, melhora } \\
\text { significativa na amplitude do ombro } \\
\text { durante o acompanhamento, baixos } \\
\text { níveis de dor, estresse e fadiga após } \\
\text { intervenção. }\end{array}$ & $\begin{array}{l}\text { O estudo demonstrou segurança e } \\
\text { viabilidade no treinamento funcional em } \\
\text { extremidade superior através do sistema } \\
\text { de RV, fornecendo elevada reabilitação e } \\
\text { treinamento intensivo nos pacientes com } \\
\text { AVC crônico. }\end{array}$ \\
\hline Saponisk,G. et al. & Comparar a segurança & adultos & Não ocorreu diferença significativa na & Apesar de 0 estudo ter utilizado a \\
\hline
\end{tabular}




\begin{tabular}{|c|c|c|c|c|}
\hline (2016) & $\begin{array}{l}\text { e eficácia da RV com } \\
\text { terapia recreativa na } \\
\text { recuperação motora } \\
\text { dos pacientes pós } \\
\text { AVC isquêmico agudo. }\end{array}$ & $\begin{array}{l}\text { sofreram um AVC } \\
\text { isquêmico (<3 meses) } \\
\text { com déficit motor leve } \\
\text { a moderado de } \\
\text { extremidade superior. }\end{array}$ & $\begin{array}{l}\text { função da mão, força de preensão, } \\
\text { desempenho motor, atividade de vida } \\
\text { diária, qualidade de vida ou de } \\
\text { movimento entre o grupo de realidade } \\
\text { virtual não imersiva e grupo de } \\
\text { reabilitação convencional. }\end{array}$ & $\begin{array}{l}\text { intervenção de RV sem fio e não } \\
\text { imersiva, a realidade é uma técnica } \\
\text { segura, mas que não gerou benefícios } \\
\text { significativos como terapia complementar } \\
\text { a reabilitação convencional ao ser } \\
\text { comparada a atividade recreativa. }\end{array}$ \\
\hline $\begin{array}{l}\text { Lee, S; Kim, Y; Lee, B. } \\
(2016)\end{array}$ & $\begin{array}{l}\text { Avaliar o efeito da RV } \\
\text { bilateral baseado no } \\
\text { treinamento de } \\
\text { extremidade superior } \\
\text { na função parética do } \\
\text { membro e força } \\
\text { muscular em } \\
\text { pacientes com AVC. }\end{array}$ & $\begin{array}{l}18 \quad \text { pacientes } \\
\text { sobreviventes de AVC } \\
(<6 \text { meses }) .\end{array}$ & $\begin{array}{l}\text { Melhoras significativas na função dos } \\
\text { membros superiores e força muscular no } \\
\text { grupo que realizou o treino bilateral de } \\
\text { extremidade superior com uso da RV. }\end{array}$ & $\begin{array}{l}\text { A RV é um método benéfico e viável para } \\
\text { a melhora da função de extremidades } \\
\text { superiores e força muscular nos } \\
\text { pacientes acometidos pelo AVC. }\end{array}$ \\
\hline
\end{tabular}

Fonte: Pesquisa autores. 


\section{DISCUSSÃO}

Com base nos artigos pesquisados, foi demonstrado que a RV fornece inúmeros benefícios aos pacientes acometidos pelo AVE, com comprometimento nos membros superiores.

No estudo de Soares et al. (2017), foi realizado um treinamento de membros superiores com três pacientes pós AVE em três dias consecutivos, utilizando um sistema de RV com a tecnologia Leap Motion Controller e o jogo Playground 3D. Através desta intervenção, eles observaram que houve uma diminuição do limiar motor dos hemisférios cerebrais proporcionando um melhor desempenho nos testes realizados, além da participação ativa dos pacientes durante o tratamento. Isso mostra a concordância com o estudo de Perez-Marcos et al. (2017), que demonstrou que os resultados obtidos nos pacientes do estudo foram satisfatórios em relação a função do membro superior acometido após AVC.

Corroborando com o estudo de Lee; Lee; Song (2018), a pesquisa de Galvão et al. (2015), também realizou uma comparação entre 27 indivíduos divididos em dois grupos, sendo que um recebia terapia convencional e outro grupo utilizou jogos do programa exergame Nintendo Wii, onde neste grupo os pacientes apresentaram melhora da função do membro superior parético, mostrando que a RV através da utilização deste programa pode gerar ganhos positivos na funcionalidade dos pacientes.

Ogun et al. (2019), demonstra em seu estudo os benefícios da RV imersiva no pós AVC, porém a tecnologia não imersiva também pode proporcionar ganhos em pacientes internados no ambiente hospitalar. Rocha (2021), utilizou um programa de RV não imersiva em quatro pacientes internados em um hospital da cidade de Porto Alegre - RS, duas vezes ao dia durante a semana e uma vez ao dia nos finais de semana, até o momento da alta hospitalar. Os resultados demonstraram que quanto menor o tempo de execução do jogo aumentava o desafio para os pacientes conforme o aumento da pontuação, além de que tal prática realizada em ambiente hospitalar proporciona aos pacientes melhora da motivação e participação.

Saposnik (2011), realizou uma meta análise com 195 pacientes de um total de 35 estudos, onde já demonstra que a RV é uma opção terapêutica já utilizada há 
alguns anos com o objetivo de recuperar a função motora dos pacientes acometidos pelo AVC. Corroborando com esse pensamento, Saposnik (2016), o método de RV foi considerada uma técnica segura, mas que não mostrou diferença significativa quando comparado às atividades recreativas realizadas.

Norousi-Gheidari et al. (2020), avaliando a eficácia clínica na recuperação funcional, observou que ocorreu melhora nas atividades de vida diárias e domínio de mobilidade. Visto que a RV é um método efetivo em outras patologias, o autor Silva et al. (2019), cita que a RV mostra efeitos positivos também em pacientes com a doença de Parkinson em um estudo de ensaio clinico onde avalia a função motora manual utilizando a RV. A amostra foi composta por 10 participantes de ambos os sexos, e obteve como resultado a melhora das habilidades motoras dos participantes, mesmo daqueles com o estágio da doença mais avançado.

A RV vem sendo um avanço tecnológico que associado à fisioterapia convencional torna um tratamento mais eficaz na recuperação sensorial e motora de pacientes pós AVC. Reafirmando esta frase o autor Cabrino (2020), realizou uma pesquisa com o objetivo de monitorar e analisar os efeitos terapêuticos da RV para membros superiores em pacientes hemiplégicos pós AVC, onde obteve resultados eficazes no aumento da amplitude de movimento e sensibilidade do membro afetado e melhora na qualidade de vida. Após dados expostos, o estudo de Lee, Kim, Lee (2016) e Oh et al. (2019), demonstram como é claro a importância da aplicabilidade da RV para a funcionalidade em pacientes com dificuldade motora de membros superiores pós AVC, pois juntamente com a fisioterapia convenvional torna 0 tratamento mais atrativo e motivador auxiliando na evolução de sua condição, sendo esta técnica uma promissora conduta para a área da saúde.

\section{CONCLUSÃO}

A utilização da RV é considerada um método viável e seguro na reabilitação dos pacientes pós AVC, além de ser um proposta alternativa que permite a maior interação do paciente durante os atendimentos.

Foi possível observar que tal método contribuiu na melhora da funcionalidade de membros superiores, força muscular, amplitude de movimento e cognição dos pacientes envolvidos nos estudos. 
Através disso, conclui-se que a RV pode ser um método cada vez mais utilizado na reabilitação, visto que ela promove variados benefícios e torna 0 atendimento mais interativo e motivador.

\section{REFERÊNCIAS}

ARAMAKI, Alberto Luiz et al. Virtual reality in the rehabilitation of patients with stroke: an integrative review. Arquivos de neuro-psiquiatria, v.77, n.4, p.268-278, 2019.

BARILLI, Elomar Christina Vieira Castilho; EBECKEN, Nelson Francisco Favilla; CUNHA, Gerson Gomes. A tecnologia de realidade virtual como recurso para formação em saúde pública à distância: uma aplicação para a aprendizagem dos procedimentos antropométricos. Ciência\& SaúdeColetiva, v.16, p.1247-1256, 2011. Brasil. Ministério da Saúde. Secretaria de Atenção à Saúde. Departamento de Ações Programáticas Estratégicas. Diretrizes de atenção à reabilitação da pessoa com acidente vascular cerebral/Ministério da Saúde, Secretaria de Atenção à Saúde, Departamento de Ações Programáticas Estratégicas - Brasília: Ministério da Saúde, 2013.

CABRINO, Juliane; SASSILOTO, Leticia; URBAN, Mayara A. Fisioterapia em reabilitação com realidade virtual no pós AVC em membro superior. 2020. Trabalho de Conclusão de Curso (TCC), Faculdade de Fisioterapia,Pontifícia Universidade Católica de Campinas, 2020.

GALVÃO, Maria Luiza Cincoetti et al. Efeito da realidade virtual na função motora do membro superior parético pós-Acidente Vascular Cerebral. Revista Neurociências, v. 23, n. 4, p. 493-498, 2015.

LEE, Myung Mo; LEE, Kyeong Jin; SONG, Chang Ho. Game-based virtual reality canoe paddling training to improve postural balance and upper extremity function: $A$ preliminary randomized controlled study of 30 patients with subacute stroke. Medical science monitor: international medical journal of experimental and clinical research, v. 24, p.2590, 2018.

LEE, Suhyun; KIM, Yumi; LEE, Byoung-Hee. Effect of virtual reality-based bilateral upper extremity training on upper extremity function after stroke: a randomized 
EDUCAÇÃO, SAÚDE

ETECNOLOGIA

26 A 28 DE OUTUBRO DE 2021

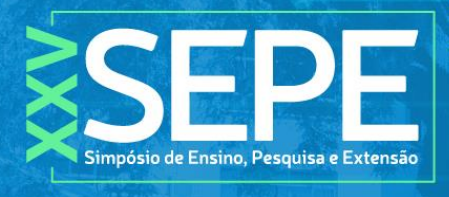

TRABALHO COMPLETO

controlled clinical trial. Occupational therapy international, v.23, n.4, p.357-368, 2016.

NOROUZI-GHEIDARI, Nahid et al. Feasibility, safety and efficacy of a virtual reality exergame system to supplement upper extremity rehabilitation post-stroke: A pilot randomized clinical trial and proof of principle. International journal of environment al research and public health, v. 17, n.1, p. 113,2020.

ÖGÜN, Muhammed Nur et al. Effect of leap motion-based 3D immersive virtual reality usage on upper extremity function in ischemic stroke patients. Arquivos de neuro-psiquiatria, v. 77, n.10, p.681-688, 2019.

$\mathrm{OH}$, Young-Bin et al. Efficacy of virtual reality combined with real instrument training forpatients with stroke: a randomized controlled trial. Archives of physical medicine and rehabilitation, v. 100, n. 8, p.1400-1408, 2019.

PEREZ-MARCOS, Daniel et al. Increasing upper limb training intensity in chronic stroke using embodied virtual reality: a pilot study. Journal of neuroengineering and rehabilitation, v. 14, n. 1, p. 1-14, 2017.

RAMOS-LIMA, Maria José Melo et al. Quality of life after stroke: impact of clinical and sociodemographic factors. Clinics, v. 73, 2018.

RANGEL, Edja Solange Souza; BELASCO, Angélica Gonçalves Silva; DICCINI, Solange. Qualidade de vida de pacientes com acidente vascular cerebral em reabilitação. Acta paulista de enfermagem, v. 26, n.2, p.205-212, 2013.

ROCHA, Gabriela Cornely. Efeitos da utilização da realidade virtual não imersiva na reabilitação de membro superior de pacientes acometidos por AVC em um hospital público de Porto Alegre. Clinical \& Biomedical Research, v. 41, n. 1, 2021.

SAPOSNIK, G., LEVIN, M. Virtual reality for stroke rehabilitation. Stroke. 2011; 42: 1380-6.

SAPOSNIK, Gustavo et al. Efficacy and safety of non-immersive virtual reality exercising in stroke rehabilitation (EVREST): a randomised, multicentre, single-blind, controlled trial. The Lancet Neurology, v. 15, n.10, p.1019-1027, 2016.

SILVA, Gabriela Letícia Oliveira et al. Repercussões do treinamento com realidade virtual não imersiva nas habilidades motoras manuais de pessoas com doença de Parkinson. Acta fisiátrica, v. 26, n. 1, p. 43-48, 2019. 
SIQUEIRA, Sandro; DEBORBA SCHNEIDERS, Paloma; GONÇALVES DA SILVA, Andréa Lúcia. Intervenções fisioterapêuticas e sua efetividade na reabilitação do paciente acometido por acidente vascular cerebral. Fisioterapia Brasil, v.20, n.4, 2019.

SOARES, Nayron Medeiros et al. Terapia baseada em realidade virtual usando o Leap Motion Controller para reabilitação do membro superior após acidente vascular cerebral. Scientia Medica, v. 27, n. 2, p. ID25935-ID25935, 2017.

World Heath Organization. (WHO). The top 10 causes of death. Genebra; 2020. Available from: https://www.who.int/news-room/fact-sheets/detail/the-top-10causes-of-death. Acesso em 08/05/2021 as 15:21. 